\title{
ON THE APPLICATION OF KNOEVENAGEL CONDENSATION FOR THE SYNTHESIS OF BENZYLIDENE BENZOTHIAZINE COMPOUNDS AND STRUCTURAL STUDY
}

\author{
Ana Maria Alves de Souza \\ Colégio de Aplicação, Universidade Federal de Pernambuco, Brasil \\ Vera Lucia de Miranda Guarda \\ Departamento de Farmácia, Universidade Federal de Ouro Preto, Brasil \\ Lúcia Fernanda Cavalcanti da Costa Leite \\ Universidade Católica de Pernambuco, Brasil \\ José Maria Barbosa Filho \\ Laboratório de Tecnologia Farmacêutica, Universidade Federal da Paraíba, Brasil \\ Maria do Carmo Alves de Lima, Suely Lins Galdino e Ivan da Rocha Pitta* \\ Departamento de Antibióticos, Universidade Federal de Pernambuco, 50670-901 Recife - PE, Brasil
}

Recebido em 22/7/05; aceito em 26/10/05; publicado na web em 6/7/06

\begin{abstract}
The synthesis and physico-chemical properties of new 6-acetylamino or 6-benzoyl-amino 2-benzylidene-4-methyl-4Hbenzo[1,4]thiazin-3-ones and 6-benzoylamino or 6-nitro 2-benzylidene-4H-benzo[1,4]thiazin-3-ones are described. These benzylidene benzothiazine compounds were prepared by the Knoevenagel condensation with benzaldehydes. The configurations and conformations of benzylidene benzothiazine derivatives were optimised using the semi-empirical method AM1.
\end{abstract}

Keywords: benzothiazines; benzylidene benzothiazines; AM1 conformational and configurational analysis.

\section{INTRODUCTION}

A large number of $2 \mathrm{H}$-benzo[1,4]thiazine derivatives and phenothiazines have been widely studied for some time now ${ }^{1-3}$. Some benzothiazines have shown microbiological and anticancerous properties $^{4-6}$. The synthesis, the physico-chemical properties and microbiological activity of some benzothiazines have also been reported by us in a previous paper ${ }^{7-11}$. The present paper describes the synthesis and the physico-chemical properties of 6-acetylamino or 6-benzoylamino 2-benzylidene-4-methyl-4H-benzo[1,4]thiazin-3-ones and 6benzoylamino or 6-nitro 2-benzylidene-4H-benzo[1,4]thiazin-3-ones resulting from Knoevenagel condensation with various benzaldehydes. Bioassay tests developed by our group have proven the ability of benzothiazine compounds to combat cocci and bacilli ${ }^{7,8,11}$. Therefore, research using improved drugs is heavily dependent on information on the structural, electronic and energy parameters of the ligants. For this reason, we are submitting a systematic semi-empirical configurational and conformational analysis of benzylidene benzothiazine derivatives $\mathbf{2 , 3}, \mathbf{7 , 9}$, and $\mathbf{1 3}$. The AM1 heat of formation for different conformations was optimised for both $E$ and $Z$ configurational isomers.

\section{SYNTHESIS AND STRUCTURAL STUDY}

2-benzylidene-6-nitro-4H-benzo[1,4]thiazin-3-one 2-3, $\mathrm{N}$-[2-(2bromo-benzylidene)-4-methyl-3-oxo-3,4-dihydro- $2 \mathrm{H}$ benzo[1,4]thiazin-6-yl]-benzamide 7, $N$-(2-benzylidene-4-methyl-3oxo-3,4-dihydro- $2 H$-benzo[1,4]thiazin-6-yl)-acetamide $\mathbf{9 , 1 0}$ and $\mathrm{N}$ [2-(4-bromo-benzylidene)-3-oxo-3,4-dihydro-2H-benzo[1,4]thiazin6-yl]-benzamide 13 can also be obtained by Knoevanagel condensation with activated methylene in compounds $\mathbf{1 , 6 , 8}$ and $\mathbf{1 2}$. The reaction takes place in the DMF in the presence of sodium methoxyde. In the benzothiazine compounds the sodium salt of the 2-amino-4nitro-benzene-thiolate is cyclised in the presence of monochloracetic acid in 6-nitro-4H-benzo[1,4]thiazin-3-one $\mathbf{1}^{7}$. $\mathrm{N}$-alkylation in position 4 , using methyl iodide in the presence of potassium hydroxide, leads to 4-methyl-6-nitro-4H-benzo[1,4]thiazin-3-one 4. The reduction of the nitro group in primary function amine with tin chloride in concentrated hydrochloric acid ${ }^{12}$ produces 6 -amino4-methyl-4H-benzo[1,4]thiazin-3-one $\mathbf{5}$ or 6-amino- $4 H$ -
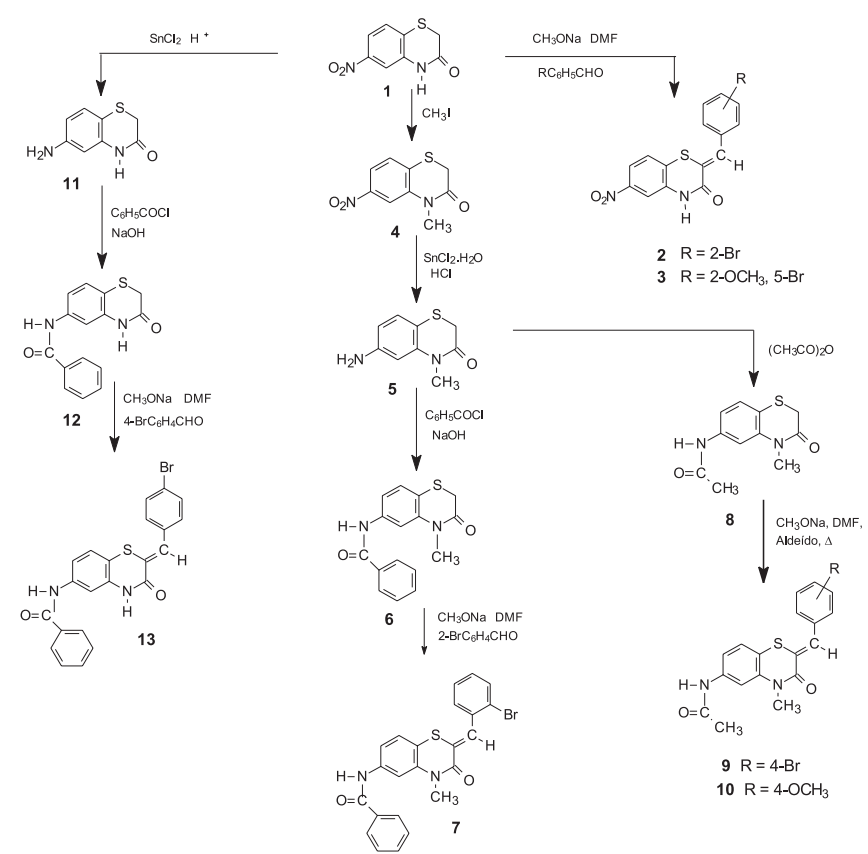

*e-mail: irpitta@gmail.com 
benzo[1,4]thiazin-3-one 11. The protection of the amino function by benzoylation or acetylation with benzoyl chloride or acetic anhydride leads to the formation of compounds $\mathbf{6 , ~ 8 , ~} 12$ (Figure 1).

The 2-benzylidene-4H-benzo[1,4]thiazin-3-ones introduced in this work were isolated in the form of a single isomer. The study using coupled spectrometry of $\mathrm{NMR}{ }^{13} \mathrm{C}-\mathrm{H}$, to determine the value of the constant of coupling between the ethylene proton and the carbon of carbonyl in position 3, when carried out for other 2benzylidene- $4 H$-benzo[1,4]thiazin-3-ones 4 -ones derivatives, showed that the principal compounds resulting from standard Knoevenagel condensation in an alkaline environment exhibit a $Z$ configuration $^{13}$.

\section{CONFORMATIONAL ANALYSIS}

The configurations and conformations of benzylidene benzothiazine derivatives $\mathbf{2}, \mathbf{3}, \mathbf{7}, \mathbf{9}$, and $\mathbf{1 3}$ (Figure 2) were optimised using the semi-empirical method AM1 in HYPERCHEM Version 7.0. For these compounds, the $Z$ configuration was the most stable in terms of energy, showing the least heat of formation. The difference between the $Z$ and $E$ isomers, based on an analysis of their most stable conformations, in terms of the heat of formation, was greater than $3.065 \mathrm{Kcal} / \mathrm{mol}$ for these compounds.

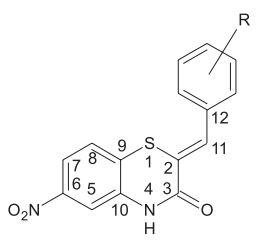

$(\mathrm{Z})$

$$
\begin{array}{ll}
2 & \mathrm{R}=2-\mathrm{Br} \\
3 & \mathrm{R}=2-\mathrm{OH}_{3}, 5-\mathrm{BR}
\end{array}
$$

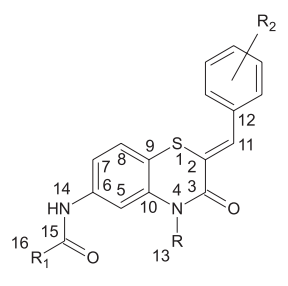

$$
\text { (Z) } \pm \mathrm{sp}
$$

$7 \mathrm{R}=\mathrm{CH}_{3} \quad \mathrm{R}_{1}=\mathrm{C}_{6} \mathrm{H}_{5} \quad \mathrm{R}_{2}=2-\mathrm{B}$

$9 \mathrm{R}=\mathrm{CH}_{3} \quad \mathrm{R}=\mathrm{CH}_{3} \quad \mathrm{R}_{2}=4-\mathrm{Br}$

$13 \mathrm{R}=\mathrm{H} \quad \mathrm{R}_{1}=\mathrm{C}_{6} \mathrm{H}_{5} \quad \mathrm{R}_{2}=4-\mathrm{Br}$
Figure 2. Benzylidene benzothiazine compounds

2-benzylidene-6-nitro-4H-benzo[1,4]thiazin-3-one derivatives 2 and 3 exhibited $2 Z 11.12 \pm s p$ conformations with a bromine atom in the ortho position near the sulphur, and the 3 Z $11.12 \pm$ ap conformations with the methoxy group opposite the sulphur atom, with little heat of formation. In the case of the $N$-[2-(2-bromobenzylidene)-4-methyl-3-oxo-3,4-dihydro- $2 H$-benzo[1,4]thiazin-6yl]-benzamide derivative 7 , the $11.12 \pm$ ap conformation proved to be the most stable. In compounds $\mathbf{7 , 9}$, and $\mathbf{1 3}$, which have an amide group in position 6 , two coplanar limit conformations were considered $6.14 \pm s p$ and $6.14 \pm a p$, with the $6.14 \pm a p$ conformers showing minor dipole moment. The $6.14+90 s c$ conformation, exhibiting the phenyl group in diagonal position in relation to the molecular plane is unstable, and generates higher heat of formation and dipole moment. Table 1 shows the values for heat of formation and dipolar moment for the more stable conformations.

\section{EXPERIMENTAL PART}

The melting points were measured on a Buchi apparatus with an oil bath, in capillary tubes. The $\mathrm{R}_{\mathrm{f}}$ in thin layer chromatography was measured on $0.2 \mathrm{~mm}$ thick silica gel plates Merck $60 \mathrm{~F}_{254}$. The IR were recorded on a Perkin-Elmer 1310 apparatus, in $\mathrm{KBr}$ tablets with a concentration of $1 \%$, the wavenumbers being expressed in $\mathrm{cm}^{-1}$. For compounds $\mathbf{2 - 3}, \mathbf{7}, \mathbf{1 1}$ and $\mathbf{1 3}$, the apparatus used was the IFS 66 Bruker. NMR spectra were recorded on an AC 200 FT (Bruker) spectrometer ${ }^{1} \mathrm{H}$ NMR at $200 \mathrm{MHz}$ and ${ }^{13} \mathrm{C}$ NMR at 50 $\mathrm{MHz}$, the chemical shifts $(\delta)$ being expressed in ppm and the coupling constants $(J)$ in Hertz $(\mathrm{Hz})$. For compounds 4, 9-11, the apparatus used was the Bruker AC $300 \mathrm{P}$. The MS, by EI 70eV, was recorded on an HP G1019A apparatus, coupled with a CPG (HP5890). For the 2, 3, 4-6, 8 compounds, the apparatus used was a Delsi-Nermag R-1010C. The intensity of the molecular peaks is given compared to the most intense peak $(\%)$.

The synthesis and the physico-chemical characteristics of 6nitro-4H-benzo[1,4]thiazin-3-one $\mathbf{1}$ compound are described in the literature ${ }^{6}$. The physicochemical and spectroscopic characteristics in IR, ${ }^{1} \mathrm{H}$ NMR and MS of the benzylidene benzothiazines prepared are described in the experimental part. Fragmentations observed and the intensity of the peaks of the isotopes on electronic impact in MS are in accordance with the structures.

\section{Condensation: general procedure}

A mixture of benzothiazine $\mathbf{1 , 8}$ or $\mathbf{1 2}(0.85 \mathrm{mmol})$ and substituted benzaldehyde $(1.01 \mathrm{mmol})$ in $5 \mathrm{~mL}$ of anhydrous DMF was treated with an excess of sodium methoxyde $(1.5 \mathrm{mmol})$. The reaction is exothermic and the mixture was maintained under agitation for 10 min at room temperature before being refluxed at $125{ }^{\circ} \mathrm{C}$ for $4 \mathrm{~h}$. After cooling, the precipitate obtained was purified using flash chromatography on silica with chloroform-hexane or washing with water, toluene or acetic acid.

\section{Amination: general procedure}

6-nitro-4H-benzo[1,4]thiazin-3-one 1 or 4-methyl-6-nitro-4Hbenzo[1,4]thiazin-3-one $4(3.5 \mathrm{mmol})$ was added in small quantities to a frozen solution containing $3.72 \mathrm{~g}$ of $\mathrm{SnCl}_{2}, 2 \mathrm{H}_{2} \mathrm{O}$ in $4 \mathrm{~mL}$ of concentrated hydrochloric acid $(36 \% \mathrm{~m} / \mathrm{m})$. The mixture was agitated for $15 \mathrm{~min}$ at room temperature and then refluxed for $2 \mathrm{~h}$. After cooling, the precipitate in the form of hydrochlorate was washed with water. To release the amine, a solution of $\mathrm{NaOH} 20 \%$ was added until $\mathrm{pH}$ reached 10 . The solid compounds were separated by filtration, washed with water and $\mathrm{NaOH} 10 \%$ before being purified by recrystallization in water.

\section{Benzoylation: general procedure}

A suspension of 6-amino-4-methyl-4H-benzo[1,4]thiazin-3-one 5 (1.55 g 5 mmol) or 6-amino-4H-benzo[1,4]thiazin-3-one $11(0.9$

Table 1. Heat of formation and dipole moment for benzylidene benzothiazine conformations

\begin{tabular}{lccc}
\hline$N^{\circ}$ & Conformations & Heat of formation Kcal/mol & Dipole moment $\mu$ \\
\hline $\mathbf{2 Z}$ & $11.12 \pm s p / 11.12 \pm a p$ & $50.638 / 52.661$ & $7.613 / 6.139$ \\
$\mathbf{3 Z}$ & $11.12 \pm a p / 11.12 \pm s p$ & $12.487 / 15.598$ & $7.475 / 5.545$ \\
$\mathbf{7 Z}$ & $11.12 \pm a p / 6.14 \pm s p / 6.14 \pm a p$ & $52.606 / 53.426$ & $6.790 / 4.215$ \\
$\mathbf{9 Z}$ & $6.14 \pm s p / 6.14 \pm a p$ & $16.008 / 16.374$ & $5.819 / 4.724$ \\
$\mathbf{1 3 Z}$ & $6.14 \pm \mathrm{sp} / 6.14 \pm a p$ & $42.439 / 42.854$ & $6.416 / 4.987$ \\
\hline
\end{tabular}


g, $5 \mathrm{mmol}$ ) in $20 \mathrm{~mL}$ of $\mathrm{NaOH} 5 \%$ was added to $2 \mathrm{~mL}$ of benzoyl chloride. The reactional mixture was maintained under strong agitation for $10 \mathrm{~min}$. The benzoyl compound was precipitated as a mass. It was separated by filtration, washed with water and purified by crystallization in $95 \%$ ethanol.

2-(2-bromo-benzylidene)-6-nitro-4H-benzo[1,4]thiazin-3-one (2)

$\mathrm{C}_{15} \mathrm{H}_{9} \mathrm{BrN}_{2} \mathrm{O}_{3} \mathrm{~S}$. M.p. $>300{ }^{\circ} \mathrm{C}$ dec. Yield \% 69. TLC benzene : ethyl acetate (8:2), $\mathrm{R}_{\mathrm{f}}$ 0.56. IR (KBr): $\bar{v} 2962,1674,1603,1516,1346$, $734 \mathrm{~cm}^{-1} .{ }^{1} \mathrm{H}$ NMR (DMSO-d $) \delta: 7.54(1 \mathrm{H}, \mathrm{C} 8, \mathrm{~d}, J=8.2 \mathrm{~Hz}), 7.36$ (1H, C3', m), 7.50 (1H, C4', m), 7.64 (1H, C5', m), 7.75 (1H, C6', m), $7.80(1 \mathrm{H}, \mathrm{C} 7, \mathrm{~d}, J=8.2 \mathrm{~Hz}), 7.83(1 \mathrm{H}, \mathrm{C} 11, \mathrm{~s}), 7.89(1 \mathrm{H}, \mathrm{C} 5, \mathrm{~d}$, $J=2,3 \mathrm{~Hz}), 11.44(1 \mathrm{H}, \mathrm{N} 4, \mathrm{~s})$. MS m/z(\%): $376\left(\mathrm{M}^{+} 2.9\right), 297$ (100), 251 (38), 210 (5.1), 89 (6.8).

2-(5-bromo-2-methoxy-benzylidene)-6-nitro-4H-benzo[1,4]thiazin3-one (3)

$\mathrm{C}_{16} \mathrm{H}_{11} \mathrm{BrN}_{2} \mathrm{O}_{4} \mathrm{~S}$. M.p. $199-201{ }^{\circ} \mathrm{C}$ dec. Yield \% 10. TLC benzene : ethyl acetate (7:3), $\mathrm{R}_{\mathrm{f}} 0.46$. IR (KBr): $\bar{v} 2923,1674,1593,1508$, 1339, $734 \mathrm{~cm}^{-1}$. ${ }^{1} \mathrm{H}$ NMR (DMSO-d $) \delta: 3.60\left(3 \mathrm{H}, \mathrm{OCH}_{3}, \mathrm{~s}\right), 7.58$ (1H, C8, d, J=9Hz), 7.58 (1H, C3', m), 7.77 (1H, C11, s), 7.75-7.82 (4H, C5,7,4',6', m), 10.95 (1H, N4, s). MS m/z(\%): $406\left(\mathrm{M}^{+} 0.63\right)$, 210(100), 181 (32.1), 122 (29.5), 95 (55.1).

\section{4-methyl-6-nitro-4H-benzo[1,4]thiazin-3-one (4)}

A mixture of 6-nitro-4H-benzo[1,4]thiazin-3-one $1(1.05 \mathrm{~g}, 5$ $\mathrm{mmol})$ in DMSO : ethanol (10:12.5 $\mathrm{mL})$ and potassium hydroxyde $(0.56 \mathrm{~g}, 10 \mathrm{mmol})$ was agitated at room temperature. Shortly afterwards (10 min), methyl iodide (1.42 g, $10 \mathrm{mmol}$ ) was added dropwise and the reactional mixture was then maintained under agitation at $50{ }^{\circ} \mathrm{C}$ for $15 \mathrm{~h}$. After cooling, the alkylated compound precipitated on addition of crushed ice. The methylated derivative was separated from the reactional mixture by filtration, and purified by recrystallization in ethanol 95\%. $\mathrm{C}_{9} \mathrm{H}_{8} \mathrm{~N}_{2} \mathrm{O}_{3}$ S. M.p. $183-5{ }^{\circ} \mathrm{C}$. Yield \% 80. TLC chloroform : ethanol (9:1), $\mathrm{R}_{\mathrm{f}}$ 0.76. IR (KBr): $\nabla$ 2940, 1690, 1510, 1340, $745 \mathrm{~cm}^{-1} .{ }^{1} \mathrm{H}$ NMR (DMSO-d 6$) \delta: 3.43(3 \mathrm{H}, \mathrm{C} 13, \mathrm{~s}), 3.66(2 \mathrm{H}$, $\mathrm{C} 11, \mathrm{~s}), 7.7(1 \mathrm{H}, \mathrm{C} 8, \mathrm{~d} J=8.4 \mathrm{~Hz}), 7.9(1 \mathrm{H}, \mathrm{C} 7, \mathrm{dd}, J=8.6$ and $2.2 \mathrm{~Hz})$, 7.98 (1H, C5, d, $J=2.4 \mathrm{~Hz}$ ). ${ }^{13} \mathrm{C}$ NMR (DMSO-d, DEPT): $\delta 29.3$ (C2), 31.5 (C13), 112.2 (C5), 117.5 (C7), 128.5 (C8), 131.9 (C9), 140.4 (C10), 146.3 (C6), 164.6 (C3). MS m/z(\%): $224\left(\mathrm{M}^{+} 100\right), 181$ (65.1), 149 (33.8), 95 (45).

6-amino-4-methyl-4H-benzo[1,4]thiazin-3-one (5)

$\mathrm{C}_{9} \mathrm{H}_{10} \mathrm{~N}_{2} \mathrm{OS}$.HCl. M.p. $228-230{ }^{\circ} \mathrm{C}, \mathrm{C}_{9} \mathrm{H}_{10} \mathrm{~N}_{2} \mathrm{OS} 131-133^{\circ} \mathrm{C}$. Yield $\%$.97. TLC chloroform : ethanol $(9: 1), \mathrm{R}_{\mathrm{f}} 0.61$. IR (KBr): $\bar{v} 3430$, $3380,1655,1610,1370,1140,800 \mathrm{~cm}^{-1} .{ }^{1} \mathrm{H}$ NMR (DMSO-d $) \delta$ : 3.25 ( $3 \mathrm{H}, \mathrm{C} 13, \mathrm{~s}), 3.31(2 \mathrm{H}, \mathrm{C} 11, \mathrm{~s}), 5.24(2 \mathrm{H}, \mathrm{N} 14, \mathrm{~s}), 6.28$ (1H, C7, dd, $J=8.2$ and $2.1 \mathrm{~Hz}), 6.46(1 \mathrm{H}, \mathrm{C} 5, \mathrm{~d}, J=2.2 \mathrm{~Hz}), 6.98(1 \mathrm{H}, \mathrm{C} 8, \mathrm{~d}$, $J=8.2 \mathrm{~Hz}$ ). ${ }^{13} \mathrm{C}$ NMR (DMSO-d, DEPT): $\delta 31.2$ (C2), 31.3 (C13), 103.6 (C5), 106.7 (C9), 109.2 (C7), 128.3 (C8), 140.8 (C10), 148.5 (C6), 165.6 (C3). MS m/z(\%): 194 (M+100), 165 (17.7), 151 (29.5), 149 (28.3), 133 (11).

N-[4-methyl-3-oxo-3,4-dihydro-2H-benzo[1,4]thiazin-6-yl]benzamide (6)

$\mathrm{C}_{16} \mathrm{H}_{14} \mathrm{~N}_{2} \mathrm{O}_{2}$ S. M.p. 185-186. Yield \% 96. TLC toluene : ethyl acetatel (6:4), $\mathrm{R}_{\mathrm{f}}$ 0.42. IR (KBr): $\bar{v} 3280,2910,1660,1640,1630$,

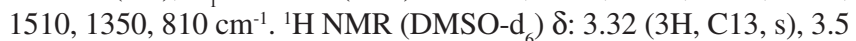
(2H, C11, s), $7.36(1 \mathrm{H}, \mathrm{C} 8, \mathrm{~d}, J=8.4 \mathrm{~Hz}), 7.53-7,57$ (4H, C7, 3', 4', ,', m), $7.76(1 \mathrm{H}, \mathrm{C} 5, \mathrm{~d}, J=2.2 \mathrm{~Hz}), 7.96\left(2 \mathrm{H}, \mathrm{C}^{\prime}, 6^{\prime}, \mathrm{m}\right), 10.33$ (1H, N14, s). ${ }^{13} \mathrm{C}$ NMR (DMSO-d, DEPT): $\delta 30.4$ (C2), 31.4 (C13), 109.8 (C5), 114.9 (C7), 116.6 (C9), 127.5 (C2',6'), 127.8 (C8), 128.3 (C3',5'), 131.6 (C4'), 134.6 (C16), 138.5 (C10 or C6), 140.1 (C10 or C6),
165.3 (C3 or $\mathrm{C} 12), 165.5$ (C3 or $\mathrm{C} 12)$. MS m/z(\%): $298\left(\mathrm{M}^{+} 37.8\right)$, 105 (100), 77 (53.9).

$\mathrm{N}$-[2-(2-bromo-benzylidene)-4-methyl-3-oxo-3,4-dihydro-2Hbenzo[1,4]thiazin-6-yl]-benzamide (7)

An equimolar mixture of N-[4-methyl-3-oxo-3,4-dihydro- $2 \mathrm{H}$ benzo[1,4]thiazin-6-yl]-benzamide 6 (150 mg, $0.5 \mathrm{mmol})$, 2-bromobenzaldehyde ( $93 \mathrm{mg}$ ) in $8 \mathrm{~mL}$ of anhydrous DMF and an excess of sodium methoxyde was agitated for 5 min before being refluxed for 4h. After cooling, the precipitate obtained was washed with water. $\mathrm{C}_{23} \mathrm{H}_{17} \mathrm{BrN}_{2} \mathrm{O}_{2}$ S. M.p. 205-210 ${ }^{\circ} \mathrm{C}$. Yield \% 60. TLC chloroform : ethanol (9:1), $\mathrm{R}_{\mathrm{f}}$ 0.66. IR (KBr): $\bar{v}$ 3271, 1647, 1599, 1304, 1149, $698 \mathrm{~cm}^{-1} .{ }^{1} \mathrm{H}$ NMR (DMSO-d $)$ \&: $3.5(3 \mathrm{H}, \mathrm{C} 13, \mathrm{~s}), 7.3(1 \mathrm{H}, \mathrm{C} 8, \mathrm{~d}$, $J=8.2 \mathrm{~Hz}$ ), 7.32-7.41 (1H, C3', m), 7.5-7.62 (6H, C4',5',6', C3",4",5", m), $7.76(1 \mathrm{H}, \mathrm{C} 7, \mathrm{~d}, J=7.8 \mathrm{~Hz}), 7.82(1 \mathrm{H}, \mathrm{C} 11, \mathrm{~s}), 7.84(1 \mathrm{H}, \mathrm{C} 5, \mathrm{~d}$, $J=2 \mathrm{~Hz}), 7.96$ (2H, C2",6", dd, $J=8,1$ and 1,6Hz), 10.45 (1H, N14, s). MS m/z(\%): 464 (M+10.2), 447 (59.9), 385 (100), 291 (60.9), 105 (45.3).

\section{N-[4-methyl-3-oxo-3,4-dihydro-2H-benzo[1,4]thiazin-6-yl]- acetamide $(\mathbf{8})$}

A suspension of 6-amino-4-methyl-4H-benzo[1,4]thiazin-3-one $5(1.55 \mathrm{~g}, 5 \mathrm{mmol})$ in $10 \mathrm{~mL}$ of acetic anhydride was refluxed for 10 min. After cooling, $10 \mathrm{~mL}$ of water was added for hydrolysis of the excess anhydride. The mixture was again refluxed for $5 \mathrm{~min}$. After cooling, the acetylated compound precipitated was washed with water and purified by recrystallization in $95 \%$ ethanol. $\mathrm{C}_{11} \mathrm{H}_{12} \mathrm{~N}_{2} \mathrm{O}_{2}$ S. M.p. 215-218 ${ }^{\circ} \mathrm{C}$. Yield \% 97. TLC chloroform : ethanol (9:1), $R_{\mathrm{f}} 0.58$. IR $(\mathrm{KBr}): \bar{v} 3320,2860,1680,1645,1590,1530,1370,820 \mathrm{~cm}^{-1} .{ }^{1} \mathrm{H}$ NMR (DMSO-d $)$ ) 2.03 (3H, C15, s), $3.28(3 \mathrm{H}, \mathrm{C} 13, \mathrm{~s}), 3.46(2 \mathrm{H}$, C2, s), 7.25-7,27 (2H, C7,8, m), $7.53(1 \mathrm{H}, \mathrm{C} 5, \mathrm{~d}, J=2.2 \mathrm{~Hz}), 10.03$ $(1 \mathrm{H}, \mathrm{N} 14, \mathrm{~s}) .{ }^{13} \mathrm{C}$ NMR (DMSO-d, DEPT): $\delta 24$ (C16), 30.4 (C2), 31.4 (C13), 108.5 (C5), 113.7 (C7), 115.8 (C9), 127.9 (C8), 138.6 (C6 or C10), 140.1 (C6 or C10), 165.3 (C3), 168.3 (C15). MS m/ z(\%): $236\left(\mathrm{M}^{+}\right.$94.9), 194 (100), 149 (38.7), 83 (29.6).

$\mathrm{N}$-[2-(4-bromo-benzylidene)-4-methyl-3-oxo-3,4-dihydro-2Hbenzo[1,4]thiazin-6-yl]-acetamide (9)

$\mathrm{C}_{18} \mathrm{H}_{15} \mathrm{BrN}_{2} \mathrm{O}_{2} \mathrm{~S}$. M.p. $239-241^{\circ} \mathrm{C}$. Yield \% 28. TLC chloroform : ethanol (9:1), $\mathrm{R}_{\mathrm{f}}$ 0.39. IR (KBr): $\nabla$ 3548, 3332, 1698, 1639, 1598,

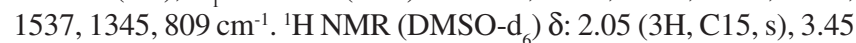
$(3 \mathrm{H}, \mathrm{C} 13, \mathrm{~s}), 7.29(1 \mathrm{H}, \mathrm{C} 8, \mathrm{~d}, J=8.4 \mathrm{~Hz}), 7.32(1 \mathrm{H}, \mathrm{C} 7, \mathrm{dd}, J=8.7$ and $1.8 \mathrm{~Hz}$ ), 7.59 (1H, C5, d, J=1.8Hz), 7.6 (2H, C2', 6', d, $J=8.4 \mathrm{~Hz}), 7.7$ (2H, C3', 5', d, J=8.7Hz), 7,76 (1H, C11, s), 10.1 (1H, N14, s). MS m/ z(\%): 402 (M+ 82.1), 362 (100), 360 (77.9), 327 (30.2), 161 (22.1).

$\mathrm{N}$-[2-(4-methoxy-benzylidene)-4-methyl-3-oxo-3,4-dihydro- $2 \mathrm{H}$ benzo[1,4]thiazin-6-yl]-acetamide (10)

$\mathrm{C}_{19} \mathrm{H}_{18} \mathrm{~N}_{2} \mathrm{O}_{3}$ S. M.p. $200-203{ }^{\circ} \mathrm{C}$. Yield \% 66. TLC chloroform : ethanol (9:1), $\mathrm{R}_{\mathrm{f}}$ 0.47. IR (KBr): $\bar{v}$ 3347, 1686, 1599, 1508, 1258, $1147 \mathrm{~cm}^{-1} .{ }^{1} \mathrm{H}$ NMR (DMSO-d $) \delta: 2.05(3 \mathrm{H}, \mathrm{C} 15, \mathrm{~s}), 3.43(3 \mathrm{H}, \mathrm{C} 13$, s), $3.82\left(3 \mathrm{H}, \mathrm{OCH}_{3}, \mathrm{~s}\right), 7.07$ (2H, C3',5', d, J=9Hz), 7.65 (2H, C2' 6', d, $J=8.7 \mathrm{~Hz}), 7.28(1 \mathrm{H}, \mathrm{C} 8, \mathrm{~d}, J=8.4 \mathrm{~Hz}), 7.32(1 \mathrm{H}, \mathrm{C} 7, \mathrm{dd}, J=8.7$ and $1.5 \mathrm{~Hz}), 7.58(1 \mathrm{H}, \mathrm{d}, \mathrm{C} 5, J=1.2 \mathrm{~Hz}), 7.75(1 \mathrm{H}, \mathrm{C} 11, \mathrm{~s}), 10.08(1 \mathrm{H}$, N14, s). MS m/z(\%): 354 (M+100), 321 (95.2), 312 (38.2), 279 (35.6), 204 (18.7).

\section{6-amino-4H-benzo[1,4]thiazin-3-one (11)}

$\mathrm{C}_{8} \mathrm{H}_{8} \mathrm{~N}_{2} \mathrm{OS}$. M.p. $225-228{ }^{\circ} \mathrm{C}$. Yield \% 91. TLC chloroform : ethanol (9:1), $\mathrm{R}_{\mathrm{f}}$ 0.38. IR (KBr): $\bar{v}$ 3442, 3349, 3201, 1678, 1623, 1380, $522 \mathrm{~cm}^{-1} .{ }^{1} \mathrm{H}$ NMR (DMSO-d $)$ ) $8: 3.3(2 \mathrm{H}, \mathrm{C} 2, \mathrm{~s}), 5.14(2 \mathrm{H}$, $\mathrm{N} 14, \mathrm{~s}), 6.21(1 \mathrm{H}, \mathrm{C} 7, \mathrm{dd}, J=9.3$ and $2.7 \mathrm{~Hz}), 6.23(1 \mathrm{H}, \mathrm{C} 5, \mathrm{~d}, J=1.5 \mathrm{~Hz})$, $6.9(1 \mathrm{H}, \mathrm{C} 8, \mathrm{~d}, J=8.4 \mathrm{~Hz}), 10.22(1 \mathrm{H}, \mathrm{N} 14, \mathrm{~s}) .{ }^{13} \mathrm{C}$ NMR (DMSO-d, 
DEPT): $\delta 29.6$ (C2), 102.5 (C7), 103.4 (C9), 109.4 (C5), 127.7 (C8), 139.1 (C10), 148.2 (C6), 165.7 (C3). MS m/z(\%): $180\left(\mathrm{M}^{+}\right.$ 100), 151 (18.1), 135 (25.5).

N-(3-oxo-3,4-dihydro-2H-benzo[1,4]thiazin-6-yl)-benzamide (12)

$\mathrm{C}_{15} \mathrm{H}_{12} \mathrm{~N}_{2} \mathrm{O}_{2} \mathrm{~S}$ M.p. $268-270{ }^{\circ} \mathrm{C}$. Yield \% 86. TLC chloroform : ethanol (9:1), $\mathrm{R}_{\mathrm{f}}$ 0.56. IR (KBr): $\bar{\nabla} 3300,3200,1675,1645,1595$, 1520, 1380, 810, $700 \mathrm{~cm}^{-1} .{ }^{1} \mathrm{H}$ NMR (DMSO-d $) \delta: 3.43(2 \mathrm{H}, \mathrm{C} 2$, s), $7.26(1 \mathrm{H}, \mathrm{C} 8, \mathrm{~d}, J=8.6 \mathrm{~Hz}), 7.33(1 \mathrm{H}, \mathrm{C} 7, \mathrm{dd}, J=8.8$ and $2 \mathrm{~Hz})$, 7.48-7.59 (4H, C5, C3', 4', 5', m), 7.92 (2H, C2', 6', dd, $J=8$ and 1,6 $\mathrm{Hz}), 10.31$ (1H, N4, s), $10.62(1 \mathrm{H}, \mathrm{N} 14, \mathrm{~s}) .{ }^{13} \mathrm{C}$ RMN (DMSO-d DEPT): $\delta 29.1$ (C2), 109.2 (C5), 113.4 (C9), 115.1 (C7), 127.2 (C3',5'), 127.6 (C8), 128.3 (C2',6'), 131.5 (C4'), 134.7 (C16), 137.6 (C10), 138.1 (C6), 165.3 (C3), 165.4 (C15).

$\mathrm{N}$-[2-(4-bromo-benzylidene)-3-oxo-3,4-dihydro- $2 \mathrm{H}$ -

benzo[1,4]thiazin-6-yl]-benzamide (13)

$\mathrm{C}_{22} \mathrm{H}_{15} \mathrm{BrN}_{2} \mathrm{O}_{2} \mathrm{~S}$ M.p. $167-168{ }^{\circ} \mathrm{C}$. Yield \% 44. TLC chloroform : ethanol (9:1), R $\mathrm{R}_{\mathrm{f}}$ 0.6. IR (KBr): $\bar{v} 3084,2927,1654,1584,1514,1341$, $1132,819 \mathrm{~cm}^{-1} .{ }^{1} \mathrm{H}$ NMR (DMSO-d $) \delta: 7.27(1 \mathrm{H}, \mathrm{C} 8, \mathrm{~d}, J=8.4 \mathrm{~Hz})$, $7.34(1 \mathrm{H}, \mathrm{C} 7, \mathrm{dd}, J=8.7$ and $2.1 \mathrm{~Hz}), 7.5-7.53\left(2 \mathrm{H}, \mathrm{C} 2{ }^{\prime}, 6^{\prime}, \mathrm{m}\right), 7.53$ $(1 \mathrm{H}, \mathrm{C} 11, \mathrm{~s}), 7.56\left(2 \mathrm{H}, \mathrm{C} 3^{\prime}, 5^{\prime}, \mathrm{d}, J=8.1 \mathrm{~Hz}\right), 7.57(1 \mathrm{H}, \mathrm{C} 5, \mathrm{~d}, J=1.5 \mathrm{~Hz})$, 7.59-7.62 (3H, C3",4",5", m), 7.93 (2H, C2",6", dd, $J=8.4$ and 1.5Hz), $10.31(1 \mathrm{H}, \mathrm{s}), 10.62(1 \mathrm{H}, \mathrm{s}) . \mathrm{MS} \mathrm{m} / \mathrm{z}(\%): 450\left(\mathrm{M}^{+} 0.1\right), 211(100)$, 209 (96.2), 181 (9.4), 102 (27.2).

\section{ACKNOWLEDGEMENTS}

The authors would like to thank CNPq (Conselho Nacional de Desenvolvimento Científico e Tecnológico, Brasil) and CAPES
(Fundação Coordenação de Aperfeiçoamento de Pessoal de Ensino Superior) for their support.

\section{REFERENCES}

1. Gupta, R. R.; Ojha, K. G.; Phenothiazines and 1,4-Benzothiazines. Chemical and Biochemical Aspects, Elsevier: Amsterdam, 1988, ch. 4.

2. Del Corona, L.; Signorelli, G.; Pinzetta, A.; Coppi, G.; Eur. J. Med. Chem. 1992, 27, 419 .

3. Macchiarulo, A.; Costantino, G.; Fringuelli, D.; Vecchiarelli, A.; Schiafella, F.; Fringuelli, R.; Bioorg. Med. Chem. 2002, 10, 3415

4. Grandolini, G.; Ambrogi, V.; Rossi, C.; Tiralti, M. C.; Tuttobello, L.; Eur. J. Med. Chem. 1986, 21, 455 .

5. Gupta, R. R.; Dev, P. K.; Sharma, M. L.; Rajoria, C. M.; Gupta, A.; Nyati, M.; Anti-Cancer Drugs 1993, 4, 589.

6. Todorov, D. K.; Ilarionova, M. V.; Gupta, R. R.; Molna, J.; Motohashi, N.; Heterocycl. Commun. 1995, 1, 153.

7. Guarda, V. L. M.; Perrissin, M.; Thomasson, F; Ximenes, E. A.; Galdino, S. L.; Pitta, I. R.; Luu-Duc, C.; Heterocycl. Commun. 2000, 6, 49.

8. Guarda, V. L. M.; Perrissin, M.; Thomasson, F.; Ximenes, E. A.; Galdino, S. L.; Pitta, I. R.; Luu-Duc, C.; Il Farmaco 2001, 56, 689.

9. Guarda, V. L. M.; Perrissin, M.; Thomasson, F.; Ximenes, E. A.; Galdino, S. L.; Pitta, I. R.; Luu-Duc, C.; Barbe J.; Eur. J. Med. Chem. 2003, 38, 769.

10. Guarda, V. L. M.; Pereira, M. A.; De Simone, C. A.; Albuquerque, J. F. C.; Galdino, S. L.; Chantegrel, J.; Perrissin, M.; Beney, C.; Thomasson, F.; Pitta, I. R.; Luu-Duc, C.; Sulfur Lett. 2003, 26, 17.

11. Guarda, V. L. M.; Perrisin, M.; Thomasson, F.; Ximenes, E. A.; Galdino, S. L.; Pitta, I. R.; Luu-Duc, C.; Heterocycl. Commun. 2004, 10, 47.

12. Cechetti, V.; Dominici, S.; Fravolini, A.; Schiaffella, F.; Eur. J. Med. Chem. 1984, 19, 29.

13. Brandão, S. S. F.; Guarda, V. L. M.; Chantegrel, J.; Perrissin, M.; Galdino, S. L.; Thomasson, F.; Souza, V. M.; Lima, M. C. A.; Leite, L. F. C. C.; Pitta, I. R.; Luu-Duc, C.; Boll. Chim. Farmac. 2000, 139, 54. 